\title{
STABILITY OF tredecic FUNCTIONAL EQUATION IN MATRIX NORMED SPACES
}

\author{
Murali Ramdoss ${ }^{1}$, Sandra Pinelas ${ }^{2}$, Vithya Veeramani ${ }^{3}$ \\ Departamento de Ciencias Exatas e Naturais, Academia Militar, 2720-113 Amadora, Portugal
}

ABSTRACT

In this current work, we define and find the general solution of the following tredecic functional equation

$$
\begin{aligned}
& f(x+7 y)-13 f(x+6 y)+78 f(x+5 y)-286 f(x+4 y)+715 f(x+3 y) \\
& -1287 f(x+2 y)+1716 f(x+y)-1716 f(x)+1287 f(x-y) \\
& -715 f(x-2 y)+286 f(x-3 y)-78 f(x-4 y)+f(x-5 y)-f(x-6 y)=13 ! f(y)
\end{aligned}
$$

where $13 !=6227020800$. We also investigate and establish the generalized Ulam-Hyers stability of this functional equation in matrix normed spaces by using the fixed point method.

\section{Introduction}

In 1940, an interesting topic was presented by S. M. Ulam [18] triggered the study of stability problems for various functional equations. He addressed a question concerning the stability of homomorphism. In the following year, 1941, D. $\mathrm{H}$. Hyers [5] was able to give a partial solution to Ulam's question. The result of Hyers was then generalized by Aoki [1] for additive mappings. In 1978, Th. M. Rassias [14] succeeded in extending the result of Hyers theorem by weakening the condition for the Cauchy difference.

The stability phenomenon that was presented by Th. M. Rassias is called the generalized Hyers-Ulam stability. In 1994, a generalization of the Rassias theorem was obtained by Gavruta [4] by replacing the unbounded Cauchy difference by a general control function. A further generalization of the Hyers-Ulam stability for a large class of mapping was obtained by Isac and Th. M. Rassias [6]. They also presented some applications in non-linear analysis, especially in fixed point theory. This terminology may also be applied to the cases of other functional equations [2, 3, 13, 15, 17, 20]. Also, the generalized Hyers-Ulam stability of functional equations and inequalities in matrix normed spaces has been studied by number of authors $[7,8,9,10,12,19]$.

K. Ravi and B. V. Senthil Kumar [16] discussed the general solution of undecic functional equation and proved the stability of this functional equation in quasi $\beta$ - normed spaces by applying the fixed point method.

In this paper, we introduce the following new functional equation

$$
\begin{aligned}
& f(x+7 y)-13 f(x+6 y)+78 f(x+5 y)-286 f(x+4 y)+715 f(x+3 y) \\
& -1287 f(x+2 y)+1716 f(x+y)-1716 f(x)+1287 f(x-y) \\
& -715 f(x-2 y)+286 f(x-3 y)-78 f(x-4 y)+f(x-5 y)-f(x-6 y)=13 ! f(y)
\end{aligned}
$$

where $13 !=6227020800$ is said to be tredecic functional equation since the function $f(x)=c x^{13}$ is its solution.

In Section 2, we study the tredecic functional equation (1).

In Section 3, using the fixed point technique, we prove the Hyers-Ulam stability of the functional equation (1) in matrix normed spaces.

\section{Tredecic Functional Equation (1)}

In this section, we study the tredecic functional equation (1). For this, let us consider $A$ and $B$ be real vector spaces.

Theorem 1 If a mapping $f: \mathrm{A} \rightarrow \mathrm{B}$ satisfies the functional equation (1) for all $x, y \in \mathrm{A}$, then $f(2 x)=2^{13} f(x)$ for all $x \in \mathrm{A}$.

Proof. Letting $x=y=0$ in (1), one gets $f(0)=0$. Replacing $x=0, y=x$ and $x=x, y=-x$ in (1) and adding the two resulting equations, we get

$$
f(-x)=-f(x)
$$

Hence, $f$ is an odd mapping. Replacing $x=0, y=2 x$ and $x=7 x, y=x$ in (1) and Subtracting Equations the two 
resulting equations, we get

$$
\begin{aligned}
& 13 f(13 x)-90 f(12 x)+286 f(11 x)-650 f(10 x)+1287 f(9 x) \\
&-1924 f(8 x)+ 1716 f(7 x)-858 f(6 x)+715 f(5 x)-858 f(4 x) \\
&+78 f(3 x)-6227020384 f(2 x)+6227020801 f(x)=0
\end{aligned}
$$

for all $x \in \mathrm{A}$. Replacing $(x, y)$ by $(6 x, x)$ in (1)and and multiplying by 13 , we get

$$
\begin{aligned}
& 13 f(13 x)-169 f(12 x)+1014 f(11 x)-3718 f(10 x)+9295 f(9 x) \\
& -16731 f(8 x)+22308 f(7 x)-22308 f(6 x)+16731 f(5 x) \\
& \quad-9295 f(4 x)+3718 f(3 x)-1014 f(2 x)+80951270230 f(x)=0
\end{aligned}
$$

for all $x \in \mathrm{A}$. Subtracting Equations (2) and (3), we arrive at

$$
\begin{aligned}
& 79 f(12 x)-728 f(11 x)+3068 f(10 x)-8008 f(9 x)+14807 f(8 x) \\
& +21450 f(6 x)-16016 f(5 x)+8437 f(4 x)-3640 f(3 x) \\
& \quad-20592 f(7 x)-6227019370 f(2 x)+87178291030 f(x)=0
\end{aligned}
$$

for all $x \in \mathrm{A}$. Replacing $(x, y)$ by $(5 x, x)$ in (1) and multiplying by 79 , we get

$$
\begin{aligned}
& 79 f(12 x)-1027 f(11 x)+6162 f(10 x)-22594 f(9 x)+56485 f(8 x) \\
& -101673 f(7 x)+135564 f(6 x)-135564 f(5 x)+101673 f(4 x) \\
& -56485 f(3 x)+22594 f(2 x)-491934649300 f(x)=0
\end{aligned}
$$

for all $x \in A$. Subtracting Equations (4) and (5), we have

$$
\begin{array}{rl}
299 f(11 x)- & 3094 f(10 x)+14586 f(9 x)-41678 f(8 x)+81081 f(7 x) \\
-114114 & f(6 x)+119548 f(5 x)-93236 f(4 x) \\
+ & 52845 f(3 x)-6227041964 f(2 x)+579112940300 f(x)=0
\end{array}
$$

for all $x \in \mathrm{A}$. Replacing $(x, y)$ by $(4 x, x)$ in (1) and multiplying by 299 , we obtain

$$
\begin{aligned}
& 299 f(11 x)-3887 f(10 x)+23322 f(9 x)-85514 f(8 x)+213785 f(7 x) \\
& -384813 f(6 x)+513084 f(5 x)-513084 f(4 x) \\
& +384813 f(3 x)-213486 f(2 x)-1861879138000 f(x)=0
\end{aligned}
$$

for all $x \in A$. Subtracting Equations (6) and (7), we obtain

$$
\begin{gathered}
793 f(10 x)-8736 f(9 x)+43836 f(8 x)-132704 f(7 x)+270699 f(6 x) \\
-393536 f(5 x)+419848 f(4 x)-6226828478 f(2 x) \\
-331968 f(3 x)+2440992078000 f(x)=0
\end{gathered}
$$

for all $x \in \mathrm{A}$. Replacing $(x, y)$ by $(3 x, x)$ in (1) and multiplying by 793 , we arrive at

$$
\begin{aligned}
793 f(10 x)-10309 f(9 x) & +61854 f(8 x)-226798 f(7 x)+566995 f(6 x) \\
-1020591 f(5 x)+1360788 f(4 x)-1359995 f(3 x) & \\
+ & 1010282 f(2 x)-4938028000000 f(x)=0
\end{aligned}
$$


for all $x \in A$. Subtracting Equations (8) and (9), we get

$$
\begin{aligned}
& 1573 f(9 x)-18018 f(8 x)+94094 f(7 x)-296296 f(6 x)+627055 f(5 x) \\
& -940940 f(4 x)+1028027 f(3 x)-6227838760 f(2 x)+7379020078000 f(x)=0
\end{aligned}
$$

for all $x \in \mathrm{A}$. Replacing $(x, y)$ by $(2 x, x)$ in (1) and multiplying by 1573 , we have

$$
\begin{aligned}
& 1573 f(9 x)-20449 f(8 x)+122694 f(7 x)-449878 f(6 x) \\
&+1124695 f(5 x)- 2022878 f(4 x)+2678819 f(3 x) \\
&- 2576574 f(2 x)-9795102144000 f(x)=0
\end{aligned}
$$

for all $x \in \mathrm{A}$. Subtracting Equations (10) and (11), we obtain

$$
\begin{array}{r}
2431 f(8 x)-28600 f(7 x)+153582 f(6 x)-497640 f(5 x)+1081938 f(4 x) \\
-1650792 f(3 x)-6225262186 f(2 x)+17174122220000 f(x)=0
\end{array}
$$

for all $x \in \mathrm{A}$. Replacing $(x, y)$ by $(x, x)$ in (1) and multiplying by 2431 , we obtain

$$
\begin{aligned}
2431 f(8 x)- & 31603 f(7 x)+189618 f(6 x)-692835 f(5 x)+1706562 f(4 x) \\
- & 2939079 f(3 x)+3476330 f(2 x)-15137890000000 f(x)=0
\end{aligned}
$$

for all $x \in \mathrm{A}$. Subtracting Equations (15) and (17), we arrive at

$$
\begin{gathered}
3003 f(7 x)-36036 f(6 x)+195195 f(5 x)-624624 f(4 x)+32312012220000 f(x) \\
+1288287 f(3 x)-6228738516 f(2 x)=0
\end{gathered}
$$

for all $x \in \mathrm{A}$. Replacing $(x, y)$ by $(0, x)$ in (1) and multiplying by 3003 , we obtain

$$
\begin{gathered}
3003 f(7 x)-36036 f(6 x)+195195 f(5 x)-624624 f(4 x)+1288287 f(3 x) \\
-1717716 f(2 x)+18699742170000 f(x)=0
\end{gathered}
$$

for all $x \in A$. Subtracting Equations (14) and (15), we get

$$
-6227020800 f(2 x)+51011754390000 f(x)=0
$$

for all $x \in \mathrm{A}$. From (16)

$$
f(2 x)=2^{13} f(x) \quad \text { forall } x \in \mathrm{A}
$$

Hence $f: \mathrm{A} \rightarrow \mathrm{B}$ is a tredecic mapping. This completes the proof.

\section{Stability of Tredecic Functional Equation in Matrix Normed Spaces}

In this section, we will investigate the Ulam-Hyers stability for the functional equation (1) in matrix normed spaces by using the fixed point method.

Throughout this section, let us consider $\left(X,\|.\|_{n}\right)$ be a matrix normed space, $\left(Y,\|.\| \|_{n}\right)$ be a matrix Banach space and let $n$ be a fixed non-negative integer.

For a mapping $f: X \rightarrow Y$, define $\mathrm{D} f: X^{2} \rightarrow Y$ and $\mathrm{D} f_{n}: M_{n}\left(X^{2}\right) \rightarrow M_{n}(Y)$ by, for all $a, b \in X$ and all $x=\left[x_{i j}\right], y=\left[y_{i j}\right] \in M_{n}(X)$.

Theorem 2 Let $l= \pm 1$ be fixed and $\psi: X^{2} \rightarrow[0, \infty)$ be a function such that there exists a $\eta<13$ with 


$$
\psi(a, b) \leq 2^{13 l} \eta \psi\left(\frac{a}{2^{l}}, \frac{b}{2^{l}}\right) \forall \mathrm{a}, \mathrm{b} \in \mathrm{X} .
$$

Let $f: X \rightarrow Y$ be a mapping satisfying

$$
\left\|\mathrm{D} f_{n}\left(\left[x_{i j}\right],\left[y_{i j}\right]\right)\right\| \leq \sum_{i, j=1}^{n} \psi\left(x_{i j}, y_{i j}\right) \forall \mathrm{x}=\left[\mathrm{x}_{\mathrm{ij}}\right], \mathrm{y}=\left[\mathrm{y}_{\mathrm{ij}}\right] \in \mathrm{M}_{\mathrm{n}}(\mathrm{X}) .
$$

Then there exists a unique tredecic mapping $\mathrm{T}: X \rightarrow Y$ such that

$$
\left\|f_{n}\left(\left[x_{i j}\right]\right)-\mathrm{T}_{n}\left(\left[y_{i j}\right]\right)\right\|_{n} \leq \sum_{i, j=1}^{n} \frac{\eta^{\frac{1-l}{2}}}{2^{13}(1-\eta)} \bar{\psi}\left(x_{i j}\right) \forall \mathrm{x}=\left[\mathrm{x}_{\mathrm{ij}}\right] \in \mathrm{M}_{\mathrm{n}}(\mathrm{X}),
$$

where $\bar{\psi}\left(x_{i j}\right)=\frac{1}{13 !}\left[\psi\left(0,2 x_{i j}\right)+\psi\left(7 x_{i j}, x_{i j}\right)+13 \psi\left(6 x_{i j}, x_{i j}\right)+79 \psi\left(5 x_{i j}, x_{i j}\right)\right.$

$$
\begin{aligned}
& +299 \psi\left(4 x_{i j}, x_{i j}\right)+793 \psi\left(3 x_{i j}, x_{i j}\right)+1573 \psi\left(2 x_{i j}, x_{i j}\right) \\
& \left.+2431 \psi\left(x_{i j}, x_{i j}\right)+3003 \psi\left(0, x_{i j}\right)\right] .
\end{aligned}
$$

Proof. Substituting $n=1$ in (19), we obtain

$$
\|\mathrm{D} f(a, b)\| \leq \psi(a, b)
$$

Replacing $(a, b)$ by $(0,2 a)$ and in $(21)$, we get

$$
\begin{aligned}
\| f(14 a)-12 f(12 a)+ & 65 f(10 a)-208 f(8 a)+429 f(6 a) \\
& -572 f(4 a)-6227020371 f(2 a) \| \leq \psi(0,2 a)
\end{aligned}
$$

for all $a \in X$. Replacing $(a, b)$ by $(7 a, a)$ in (21), we obtain

$$
\begin{aligned}
& \| f(14 a)-13 f(13 a)+78 f(12 a)-286 f(11 a)+715 f(10 a) \\
& -1287 f(9 a)+1716 f(8 a)-1716 f(7 a)+1287 f(6 a) \\
& \quad-715 f(5 a)+286 f(4 a)-78 f(3 a)+13 f(2 a)-6227020801 f(a) \| \leq \psi(7 a, a)
\end{aligned}
$$

for all $a \in X$. It follows from (22) and (23), we arrive at

$$
\begin{aligned}
& \| 13 f(13 a)-90 f(12 a)+286 f(11 a)-650 f(10 a)+1287 f(9 a) \\
& -1924 f(8 a)+1716 f(7 a)-858 f(6 a)+715 f(5 a)-858 f(4 a) \\
& \quad+78 f(3 a)-6227020384 f(2 a)+6227020801 f(a) \| \leq \psi(0,2 a)+\psi(7 a, a)
\end{aligned}
$$

for all $a \in X$. Replacing $(a, b)$ by $(6 a, a)$ in $(21)$ and multiplying by 13 , we get

$$
\begin{aligned}
& \| 13 f(13 a)-169 f(12 a)+1014 f(11 a)-3718 f(10 a)+9295 f(9 a) \\
& -16731 f(8 a)+22308 f(7 a)-22308 f(6 a)+16731 f(5 a) \\
& \quad-9295 f(4 a)+3718 f(3 a)-1014 f(2 a)+80951270230 f(a) \| \leq 13 \psi(6 a, a)
\end{aligned}
$$

for all $a \in X$. It follows from (24) and (25), we arrive at

$$
\mid 79 f(12 a)-728 f(11 a)+3068 f(10 a)-8008 f(9 a)+14807 f(8 a)-20592 f(7 a)
$$




$$
\begin{aligned}
& +21450 f(6 a)-16016 f(5 a)+8437 f(4 a)-3640 f(3 a) \\
& \quad-6227019370 f(2 a)+87178291030 f(a) \| \leq \psi(0,2 a)+\psi(7 a, a)+13 \psi(6 a, a)
\end{aligned}
$$

for all $a \in X$. Replacing $(a, b)$ by $(5 a, a)$ in (21) and multiplying by 79 , we get

$$
\begin{aligned}
& \| 79 f(12 a)-1027 f(11 a)+6162 f(10 a)-22594 f(9 a)+56485 f(8 a) \\
& -101673 f(7 a)+135564 f(6 a)-135564 f(5 a)+101673 f(4 a) \\
& \quad-56485 f(3 a)+22594 f(2 a)-491934649300 f(a) \| \leq 79 \psi(5 a, a)
\end{aligned}
$$

for all $a \in X$. It follows from (26) and (27), we arrive at

$$
\begin{aligned}
& \| 299 f(11 a)-3094 f(10 a)+14586 f(9 a)-41678 f(8 a)+81081 f(7 a) \\
& -114114 f(6 a)+119548 f(5 a)-93236 f(4 a)-6227041964 f(2 a)+52845 f(3 a) \\
& \quad+579112940300 f(a) \| \leq \psi(0,2 a)+\psi(7 a, a)+13 \psi(6 a, a)+79 \psi(5 a, a)
\end{aligned}
$$

for all $a \in X$. Replacing $(a, b)$ by $(4 a, a)$ in (21) and multiplying by 299 , we get

$$
\begin{aligned}
& \| 299 f(11 a)-3887 f(10 a)+23322 f(9 a)-85514 f(8 a)+213785 f(7 a) \\
& -384813 f(6 a)+513084 f(5 a)-513084 f(4 a) \\
& \quad+384813 f(3 a)-213486 f(2 a)-1861879138000 f(a) \| \leq 299 \psi(4 a, a)
\end{aligned}
$$

for all $a \in X$. It follows from (28) and (29), we obtain

$$
\begin{aligned}
& \| 793 f(10 a)-8736 f(9 a)+43836 f(8 a)-132704 f(7 a)+270699 f(6 a) \\
& -393536 f(5 a)+419848 f(4 a)-6226828478 f(2 a)+2440992078000 f(a) \\
& \quad-331968 f(3 a) \| \leq \psi(0,2 a)+\psi(7 a, a)+13 \psi(6 a, a)+79 \psi(5 a, a)+299 \psi(4 a, a)
\end{aligned}
$$

for all $a \in X$. Replacing $(a, b)$ by $(3 a, a)$ in (21) and multiplying by 793 , we arrive at

$$
\begin{aligned}
& \| 793 f(10 a)-10309 f(9 a)+61854 f(8 a)-226798 f(7 a)+566995 f(6 a) \\
& -1020591 f(5 a)+1360788 f(4 a)-1359995 f(3 a) \\
& +1010282 f(2 a)-4938028000000 f(a) \| \leq 793 \psi(3 a, a)
\end{aligned}
$$

for all $a \in X$. It follows from (30) and (31), we get

$$
\begin{aligned}
& \| 1573 f(9 a)-18018 f(8 a)+94094 f(7 a)-296296 f(6 a)+627055 f(5 a) \\
& -940940 f(4 a)+1028027 f(3 a)-6227838760 f(2 a)+7379020078000 f(a) \| \\
& \leq \psi(0,2 a)+\psi(7 a, a)+13 \psi(6 a, a)+79 \psi(5 a, a)+299 \psi(4 a, a)+793 \psi(3 a, a)
\end{aligned}
$$

for all $a \in X$. Replacing $(a, b)$ by $(2 a, a)$ in $(21)$ and multiplying by 1573 , we arrive at

$$
\begin{aligned}
& \| 1573 f(9 a)-20449 f(8 a)+122694 f(7 a)-449878 f(6 a) \\
& +1124695 f(5 a)-2022878 f(4 a)+2678819 f(3 a)
\end{aligned}
$$




$$
-2576574 f(2 a)-9795102144000 f(a) \| \leq 1573 \psi(2 a, a)
$$

for all $a \in X$. It follows from (32) and (33), we obtain

$$
\begin{gathered}
\| 2431 f(8 a)-28600 f(7 a)+153582 f(6 a)-497640 f(5 a)+1081938 f(4 a) \\
-1650792 f(3 a)-6225262186 f(2 a)+17174122220000 f(a) \| \\
\leq \psi(0,2 a)+\psi(7 a, a)+13 \psi(6 a, a)+79 \psi(5 a, a)+299 \psi(4 a, a) \\
+793 \psi(3 a, a)+1573 \psi(2 a, a)
\end{gathered}
$$

for all $a \in X$. Replacing $(a, b)$ by $(a, a)$ in $(21)$ and multiplying by 2431 , we obtain

$$
\begin{aligned}
& \| 2431 f(8 a)-31603 f(7 a)+189618 f(6 a)-692835 f(5 a)+1706562 f(4 a) \\
& -2939079 f(3 a)+3476330 f(2 a)-15137890000000 f(a) \| \leq 2431 \psi(a, a)
\end{aligned}
$$

for all $a \in X$. It follows from (37) and (35), we arrive at

$$
\begin{aligned}
\| 3003 f(7 a)-36036 f(6 a) & +195195 f(5 a)-624624 f(4 a)+32312012220000 f(a) \\
+ & 1288287 f(3 a)-6228738516 f(2 a) \| \\
\leq \psi(0,2 a)+\psi(7 a, a)+ & 13 \psi(6 a, a)+79 \psi(5 a, a)+299 \psi(4 a, a) \\
+ & 793 \psi(3 a, a)+1573 \psi(2 a, a)+2431 \psi(a, a)
\end{aligned}
$$

for all $a \in X$. Replacing $(a, b)$ by $(0, a)$ in $(21)$ and multiplying by 3003 , we obtain

$$
\begin{gathered}
\| 3003 f(7 a)-36036 f(6 a)+195195 f(5 a)-624624 f(4 a)+1288287 f(3 a) \\
-1717716 f(2 a)+18699742170000 f(a) \| \leq 3003 \psi(0, a)
\end{gathered}
$$

for all $a \in X$. It follows from (36) and (37), we get

$$
\begin{aligned}
& \|-6227020800 f(2 a)+51011754390000 f(a)\| \\
& \leq 4 \psi(0,2 a)+\psi(7 a, a)+13 \psi(6 a, a)+79 \psi(5 a, a)+299 \psi(4 a, a) \\
& +793 \psi(3 a, a)+1573 \psi(2 a, a)+2431 \psi(a, a)+3003 \psi(0, a) \\
& \text { for all } a \in X \text {. From (38) } \\
& \left\|-f(2 a)+2^{13} f(a)\right\| \leq \frac{1}{13 !}[\psi(0,2 a))+\psi(7 a, a)+13 \psi(6 a, a)+79 \psi(5 a, a) \\
& +299 \psi(4 a, a)+793 \psi(3 a, a)+1573 \psi(2 a, a) \\
& +2431 \psi(a, a)+3003 \psi(0, a)]
\end{aligned}
$$

Therefore,

$$
\left\|f(2 a)-2^{13} f(a)\right\| \leq \bar{\psi}(a) \forall \mathrm{a} \in \mathrm{X}
$$

Thus 


$$
\left\|f(a)-\frac{1}{2^{13 l}} f\left(2^{l} a\right)\right\| \leq \frac{\eta^{\left(\frac{1-l}{2}\right)}}{2^{13}} \bar{\psi}(a) \quad \forall \mathrm{a} \in \mathrm{X} .
$$

We consider the set $\mathrm{M}=\{f: X \rightarrow Y\}$ and introduce the generalized metric $\rho$ on $\mathrm{M}$ as follows:

$$
\rho(f, g)=\inf \left\{\mu \in \mathrm{R}_{+}:\|f(a)-g(a)\| \leq \mu \bar{\psi}(a), \forall a \in X\right\},
$$

It is easy to check that $(\mathrm{M}, \rho)$ is a complete generalized metric (see also [11]). Define the mapping $\mathrm{P}: \mathrm{M} \rightarrow \mathrm{M}$ by

$$
\mathrm{P} f(a)=\frac{1}{2^{13 l}} f\left(2^{l} a\right) \quad \forall \mathrm{f} \in \text { Manda } \in \mathrm{X} .
$$

Let $f, g \in \mathrm{M}$ and $v$ be an arbitrary constant with $\rho(f, g)=v$. Then

$$
\|f(a)-g(a)\| \leq v \bar{\psi}(a) \text { for all } a \in X .
$$

Utilizing (18), we find that

$$
\|\mathrm{P} f(a)-\mathrm{P} g(a)\|=\left\|\frac{1}{2^{13 l}} f\left(2^{l} a\right)-\frac{1}{2^{13 l}} g\left(2^{l} a\right)\right\| \leq \eta v \bar{\psi}(a) \text { for all } a \in X .
$$

Hence it holds that $\rho(\mathrm{P} f, \mathrm{P} g) \leq \eta v$, that is, $\rho(\mathrm{P} f, \mathrm{P} g) \leq \eta \rho(f, g)$ for all $f, g \in \mathrm{M}$.

It follows from (41) that $\rho(f, \mathrm{P} f) \leq \frac{\eta^{\left(\frac{1-l}{2}\right)}}{2^{13}}$.

Therefore according to Theorem 2.2 in [3], there exists a mapping $\mathrm{T}: X \rightarrow Y$ which satisfying:

1. $\mathrm{T}$ is a unique fixed point of $\mathrm{P}$ in the set $\mathrm{S}=\{g \in \mathrm{M}: \rho(f, g)<\infty\}$, which is satisfied

$$
\mathrm{T}\left(2^{l} a\right)=2^{13 l} \mathrm{~T}(a) \quad \forall \mathrm{a} \in \mathrm{X} .
$$

In other words, there exists a $\mu$ satisfying

$$
\|f(a)-g(a)\| \leq \mu \bar{\psi}(a) \forall \mathrm{a} \in \mathrm{X} .
$$

2. $\rho\left(\mathrm{P}^{k} f, \mathrm{~T}\right) \rightarrow 0$ as $k \rightarrow \infty$. This implies that

$$
\lim _{k \rightarrow \infty} \frac{1}{2^{13 k l}} f\left(2^{k l} a\right)=\mathrm{T}(a) \quad \forall \mathrm{a} \in \mathrm{X} .
$$

3. $\rho(f, \mathrm{~T}) \leq \frac{1}{1-\eta} \rho(f, \mathrm{P} f)$, which implies the inequality $\rho(f, \mathrm{~T}) \leq \frac{\eta^{\frac{1-l}{2}}}{2^{13}(1-\eta)}$.

$$
\text { So }\|f(a)-\mathrm{T}(a)\| \leq \frac{\eta^{\frac{1-l}{2}}}{2^{13}(1-\eta)} \bar{\psi}(a) \quad \forall \mathrm{a} \in \mathrm{X} \text {. }
$$

It follows from (18) and (19) that

$$
\|\mathrm{DT}(a, b)\|=\lim _{k \rightarrow \infty} \frac{1}{2^{13 k l}}\left\|\mathrm{D} f\left(2^{k l} a, 2^{k l} b\right)\right\|
$$




$$
\leq \lim _{k \rightarrow \infty} \frac{1}{2^{13 k l}} \psi\left(2^{k l} a, 2^{k l} b\right) \leq \lim _{k \rightarrow \infty} \frac{2^{k l} \eta^{k}}{2^{13 k l}} \psi(a, b)=0
$$

for all $a, b \in X$. Hence

for all $a, b \in X$. Therefore, the mapping $\mathrm{\top}: X \rightarrow Y$ is tredecic mapping. By Lemma 2.1 in [9] and (43),

$$
\begin{aligned}
& \left\|f_{n}\left(\left[x_{i j}\right]\right)-\mathrm{T}_{n}\left(\left[x_{i j}\right]\right)\right\| \leq \sum_{i, j=1}^{n}\left\|f\left(x_{i j}\right)-\mathrm{T}\left(x_{i j}\right)\right\| \\
& \leq \sum_{i, j=1}^{n} \frac{\eta^{\frac{1-l}{2}}}{2^{13}(1-\eta)} \bar{\psi}\left(x_{i j}\right) \forall \mathrm{x}=\left[\mathrm{x}_{\mathrm{ij}}\right] \in \mathrm{M}_{\mathrm{n}}(\mathrm{X}),
\end{aligned}
$$

where $\bar{\psi}\left(x_{i j}\right)=\frac{1}{13 !}\left[\psi\left(0,2 x_{i j}\right)+\psi\left(7 x_{i j}, x_{i j}\right)+13 \psi\left(6 x_{i j}, x_{i j}\right)+79 \psi\left(5 x_{i j}, x_{i j}\right)\right.$

$$
\begin{aligned}
& +299 \psi\left(4 x_{i j}, x_{i j}\right)+793 \psi\left(3 x_{i j}, x_{i j}\right)+1573 \psi\left(2 x_{i j}, x_{i j}\right) \\
& \left.+2431 \psi\left(x_{i j}, x_{i j}\right)+3003 \psi\left(0, x_{i j}\right)\right]
\end{aligned}
$$

Thus $\mathrm{T}: X \rightarrow Y$ is a unique tredecic mapping satisfying (20).

Corollary 1 Let $l= \pm 1$ be fixed and let $t, \vartheta$ be positive real numbers with $t \neq 13$. Let $f: X \rightarrow Y$ be a mapping such that

$$
\left\|\mathrm{D} f_{n}\left(\left[x_{i j}\right],\left[y_{i j}\right]\right)\right\|_{n} \leq \sum_{i, j=1}^{n} \vartheta\left(\left\|x_{i j}\right\|^{t}+\left\|y_{i j}\right\|^{t}\right) \forall \mathrm{x}=\left[\mathrm{x}_{\mathrm{ij}}\right], \mathrm{y}=\left[\mathrm{y}_{\mathrm{ij}}\right] \in \mathrm{M}_{\mathrm{n}}(\mathrm{X}) .
$$

Then there exists a unique tredecic mapping $\mathrm{T}: X \rightarrow Y$ such that

$$
\left\|f_{n}\left(\left[x_{i j}\right]\right)-\mathrm{T}_{n}\left(\left[x_{i j}\right]\right)\right\|_{n} \leq \sum_{i, j=1}^{n} \frac{\vartheta_{s}}{l\left(2^{13}-2^{t}\right)}\left\|x_{i j}\right\|^{t} \forall x=\left[x_{i j}\right] \in M_{n}(X),
$$

where $\vartheta_{s}=\frac{\vartheta}{13 !}\left[3003+2432\left(2^{t}\right)+1573\left(3^{t}\right)+793\left(4^{t}\right)+299\left(5^{t}\right)+79\left(6^{t}\right)+13\left(7^{t}\right)+8^{t}\right]$

Proof. The proof follows from Theorem 2 by taking $\psi(a, b)=\vartheta\left(\|a\|^{t}+\|b\|^{t}\right)$ for all $a, b \in X$. Then we can choose $\eta=2^{l(t-13)}$, and we can obtain the required result.

\section{Example 3 Let $\psi: \mathrm{R} \rightarrow \mathrm{R}$ be a function defined by}

$$
\psi(x)=\left\{\begin{array}{l}
\vartheta x^{13}, \quad \text { if }|x|<1 \\
\vartheta, \quad \text { otherwise }
\end{array}\right.
$$

where $\vartheta>0$ is a constant, and define a function $f: \mathrm{R} \rightarrow \mathrm{R}$ by

$$
f(x)=\sum_{n=0}^{\infty} \frac{\psi\left(2^{n} x\right)}{2^{13 n}}
$$

for all $x \in \mathrm{R}$. Then $f$ satisfies the inequality

$$
\| f(x+7 y)-13 f(x+6 y)+78 f(x+5 y)-286 f(x+4 y)+715 f(x+3 y)
$$




$$
\begin{aligned}
& -1287 f(x+2 y)+1716 f(x+y)-1716 f(x)+1287 f(x-y) \\
& -715 f(x-2 y)+286 f(x-3 y)-78 f(x-4 y)+f(x-5 y) \\
& -f(x-6 y)-13 ! f(y) \| \leq \frac{(6227028992) \vartheta}{8191}(8192)^{2}\left(|x|^{13}+|y|^{13}\right)
\end{aligned}
$$

for all $x, y \in \mathrm{R}$. Then there do not exists a tredecic mapping $\mathrm{T}: \mathrm{R} \rightarrow \mathrm{R}$ and a constant $\lambda>0$ such that

$$
|f(x)-\mathrm{T}(x)| \leq \lambda|x|^{13} \quad \forall \mathrm{x} \in \mathrm{R}
$$

Proof. It is easy to see that $f$ is bounded by $\frac{8192 \vartheta}{8191}$ on $\mathrm{R}$.

Next we have to show that $f$ satisfies (45).

If $x=y=0$, then (45) is trivial.

If $|x|^{13}+|y|^{13} \geq \frac{1}{2^{13}}$, then L.H.S of (45) is less than $\frac{(6227028992)(8192) \vartheta}{8191}$.

Suppose that $0<|x|^{13}+|y|^{13}<\frac{1}{2^{13}}$, then there exists a non-negative integer $k$ such that

$$
\frac{1}{2^{13(k+1)}} \leq|x|^{13}+|y|^{13}<\frac{1}{2^{13 k}},
$$

so that $2^{13(k-1)} x^{13}<\frac{1}{2^{13}}, 2^{13(k-1)} y^{13}<\frac{1}{2^{13}}$, and

$$
2^{n}(x), 2^{n}(y), 2^{n}(x+7 y), 2^{n}(x+6 y), 2^{n}(x+5 y), 2^{n}(x+4 y), \quad 2^{n}(x+3 y), 2^{n}(x+2 y), 2^{n}(x+y), 2^{n}(x-y), 2^{n}
$$

for all $n=0,1,2, \ldots, k-1$. Hence

$$
\begin{aligned}
& \psi\left(2^{n}(x+7 y)\right)-13 \psi\left(2^{n}(x+6 y)\right)+78 \psi\left(2^{n}(x+5 y)\right)-286 \psi\left(2^{n}(x+4 y)\right) \\
& +715 \psi\left(2^{n}(x+3 y)\right)-1287 \psi\left(2^{n}(x+2 y)\right)+1716 \psi\left(2^{n}(x+y)\right) \\
& -1716 \psi\left(2^{n} x\right)+1287 \psi\left(2^{n}(x-y)\right)-715 \psi\left(2^{n}(x-2 y)\right)+286 \psi\left(2^{n}(x-3 y)\right) \\
& -78 \psi\left(2^{n}(x-4 y)\right)+\psi\left(2^{n}(x-5 y)\right)-\psi\left(2^{n}(x-6 y)\right)-13 ! \psi\left(2^{n} y\right)=0
\end{aligned}
$$

for $n=0,1,2, \ldots, k-1$. From the definition of $f$ and (47), we obtain that

$$
\begin{aligned}
& \mid f(x+7 y)-13 f(x+6 y)+78 f(x+5 y)-286 f(x+4 y)+715 f(x+3 y) \\
& -1287 f(x+2 y)+1716 f(x+y)-1716 f(x)+1287 f(x-y)-715 f(x-2 y) \\
& +286 f(x-3 y)-78 f(x-4 y)+f(x-5 y)-f(x-6 y)-13 ! f(y) \mid \\
& \leq \sum_{n=0}^{\infty} \frac{1}{2^{13 n}} \mid \psi\left(2^{n}(x+7 y)\right)-13 \psi\left(2^{n}(x+6 y)\right)+78 \psi\left(2^{n}(x+5 y)\right) \\
& \quad-286 \psi\left(2^{n}(x+4 y)\right)+715 \psi\left(2^{n}(x+3 y)\right)-1287 \psi\left(2^{n}(x+2 y)\right) \\
& \quad+1716 \psi\left(2^{n}(x+y)\right)-1716 \psi\left(2^{n} x\right)+1287 \psi\left(2^{n}(x-y)\right)
\end{aligned}
$$




$$
\begin{aligned}
& -715 \psi\left(2^{n}(x-2 y)\right)+286 \psi\left(2^{n}(x-3 y)\right)-78 \psi\left(2^{n}(x-4 y)\right) \\
& +\psi\left(2^{n}(x-5 y)\right)-\psi\left(2^{n}(x-6 y)\right)-13 ! \psi\left(2^{n} y\right) \mid \\
\leq & \sum_{n=k}^{\infty} \frac{(6227028992) \vartheta}{2^{13 n}}=\frac{(8192)(6227028992) \vartheta}{2^{13 k} 8191} \\
\leq & \frac{(6227028992)}{8191}(8192)^{2} \vartheta\left(|x|^{13}+|y|^{13}\right) .
\end{aligned}
$$

Thus $f$ satisfies (45) for all $x, y, z \in \mathrm{R}$ with $0<|x|^{13}+|y|^{13}<\frac{1}{2^{13}}$.

Now, we claim that the tredecic functional equation (1) is not stable for $t=13$ in corollary 1 . Suppose that there exists a tredecic mapping $\mathrm{T}: \mathrm{R} \rightarrow \mathrm{R}$ and a constant $\lambda>0$ satisfying (46). Then there exists a constant $c \in \mathrm{R}$ such that $\mathrm{T}(x)=c x^{19}$ for any $x \in \mathrm{R}$. Thus we obtain the following inequality

$$
|f(x)| \leq(\lambda+|c|)|x|^{13}
$$

Let $m \in \mathrm{N}$ with $m \varepsilon>\lambda+|c|$. If $x \in\left(0, \frac{1}{2^{m-1}}\right)$, then $2^{n} x \in(0,1)$ for all $n=0,1,2, \ldots, m-1$, and for this case we get

$$
f(x)=\sum_{n=0}^{\infty} \frac{\psi\left(2^{n} x\right)}{2^{13 n}} \geq \sum_{n=0}^{m-1} \frac{\vartheta\left(2^{n} x\right)^{13}}{2^{13 n}}=m \vartheta x^{13}>(\lambda+|c|)|x|^{13}
$$

which is a contradiction to (48). Thus the tredecic functional equation (1) is not stable for $t=13$.

\section{Conclusion}

In this investigation, we identified a general solution of tredecic functional equation and establised the generalized Ulam Hyers stability of this functional equation in matrix normed spaces by using the fixed point method and also provided an example for non-stability.

\section{References}

[1] T. Aoki , On the Stability of the Linear Transformation in Banach Spaces, J. Math. Soc. Japan, 2 (1950), 64-66.

[2] M. Arunkumar, A. Bodaghi, J. M. Rassias and Elumalai Sathiya, The General Solution and Approximations of a Decic Type Functional Equation in Various Normed Spaces, Journal of the Chungcheong Mathematical Society, 29(2), (2016), 287-328.

[3] L. Cadariu and V. Radu, Fixed Points and the Stability of Jensen's Functional Equation., J. Inequal. Pure Appl. Math., 4(1) (2003), 1-7.

[4] P. Gavruta, A Generalization of the Hyers-Ulam Rassias Stability of Approximately Additive Mappings, J. Math. Anal. Appl., 184 (1994), 431-436.

[5] D. H. Hyers, On the Stability of the Linear Functional Equation, Proc. Natl. Acad. Sci. USA, 27 (1941), $222-224$.

[6] G. Isac and Th. M. Rassias, Stability of $\varphi$-Additive Mappings: Applications to Nonlinear Analysis, J. Funct. Anal., 19 (1996), 219-228.

[7] J. Lee, D. Shin and C. Park, An Additive Functional Inequality in Matrix Normed Spaces, Mathematical Inequalities and Applications, 16(4)(2013), 1009-1022.

[8] J. Lee, D. Shin and C. Park, An AQCQ- Functional Equation in Matrix Normed Spaces, Result. Math., 64 2013:146, 115.

[9] J. Lee, D. Shin and C. Park, Hyers-Ulam Stability of Functional Equations in Matrix Normed Spaces, Journal of Inequalities and Applications, 2013:22, 1-11.

[10] J. Lee, C. Park and D. Shin, Functional Equations in Matrix Normed Spaces, Proc. Indian Acad. sci., 125(3) (2015), 399-412.

[11] D. Mihet and V. Radu, On the Stability of the Additive Cauchy Functional Equation in Random Normed Spaces, J. 
Math. Anal. Appl., 343 (2008), 567-572.

[12] R. Murali and V. Vithya, Hyers-Ulam-Rassias Stability of Functional Equations in Matrix Normed Spaces: A fixed point approach, Assian Journal of Mathematics and Computer Research, 4(3) (2015), 155-163.

[13] C. Park, Stability of an AQCQ-Functional Equation in Paranormed Spaces, Advances in Difference Equations., 2012:148, 1-20.

[14] Th. M. Rassias, On the Stability of the Linear Mapping in Banach Spaces, Proc. Am. Math. Soc., 72 (1978), $297-300$.

[15] J. M. Rassias and Mohammad Eslamian, Fixed Points and Stability of Nonic Functional Equation in Quasi $\beta$ Normed Spaces, Contemporary Anal. Appl. Math., 3(2) (2015), 293-309.

[16] K. Ravi, J.M. Rassias and B.V. Senthil Kumar, Ulam-Hyers Stability of Undecic Functional Equation in Quasi- $\beta$ Normed Spaces: Fixed Point Method, Tbilisi Mathematical Science, 9(2) (2016), 83-103.

[17] Y. Shen, W. Chen, On the Stability of Septic and Octic Functional Equations, J. Computational Analysis and Applications, 18(2), (2015), 277-290.

[18] S. M. Ulam, Problems in Modern Mathematics, Science Editions, Wiley, NewYork (1964).

[19] Z. Wang and P. K. Sahoo, Stability of an ACQ- Functional Equation in Various Matrix Normed Spaces, J. Nonlinear Sci. Appl., 8 (2015), 64-85.

[20] T. Z. Xu , J.M. Rassias, M. J. Rassias, and W. X. Xu, A Fixed Point Approach to the Stability of Quintic and Sextic Functional Equations in Quasi- $\beta$ Normed Spaces. Journal of Inequalities and Applications, (2010), 1-23.

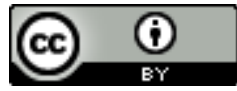

This work is licensed under a Creative Commons Attribution 4.0 International License. 